\title{
Decomposing economic disparities in risky sexual behaviors among people who inject drugs in Tehran: Blinder-Oaxaca decomposition analysis
}

\author{
Mehdi Noroozi ${ }^{1}$, Hamid Sharifi ${ }^{2}$, Alireza Noroozi ${ }^{3,4}$, Fatemah Rezaei ${ }^{5}$, Mohammad Rafi Bazrafshan ${ }^{6}$, \\ Bahram Armoon ${ }^{7}$ \\ 'Social Determinants of Health Research Center, University of Social Welfare and Rehabilitation Sciences, Tehran, Iran; ${ }^{2} \mathrm{HIV} / \mathrm{STI}$ Surveillance \\ Research Center, WHO Collaborating Center for HIV Surveillance, Institute for Future Studies in Health, Kerman University of Medical Sciences, \\ Kerman, Iran; ${ }^{3}$ rranian National Center for Addiction Studies, Tehran University of Medical Sciences, Tehran, Iran; ${ }^{4}$ Department of Neuroscience \\ and Addiction Studies, School of Advanced Technologies in Medicine, Tehran University of Medical Sciences, Tehran, Iran; ${ }^{5}$ Department of \\ Social Medicine, School of Medicine, Jahrom University of Medical Sciences, Jahrom, Iran; ${ }^{6}$ Department of Nursing, School of Nursing, Larestan \\ University of Medical Sciences, Larestan, Iran; ${ }^{7}$ Student Research Committee, School of Health, Shahid Beheshti University of Medical Sciences, \\ Tehran, Iran
}

OBJECTIVES: To our knowledge, no previous study has systematically assessed the role of economic status in risky sexual behavior among people who inject drugs (PWID) in Iran. In this study, we used Blinder-Oaxaca (BO) decomposition to explore the contribution of economic status to inequality in unprotected sex among PWID in Tehran and to decompose it into its determinants.

METHODS: Behavioral surveys among PWID were conducted in Tehran, the capital city of Iran, from November 2016 to April 2017. We employed a cross-sectional design and snowball sampling methodology. We constructed the asset index (weighted by the first principal component analysis factor) using socioeconomic data and then divided the variable into 3 tertiles. We used the BO method to decompose the economic inequality in unprotected sex.

RESULTS: Of the 520 recruited individuals, 20 were missing data for variables used to define their economic status, and were therefore excluded from the analysis. Not having access to harm reduction programs was the largest factor contributing to the economic disparity in unprotected sex, accounting for 5.5 percentage points of the $21.4 \%$ discrepancy. Of the unadjusted total economic disparity in unprotected sex, $52 \%$ was unexplained by observable characteristics included in the regression model. The difference in the prevalence of unprotected sex between the high-income and low-income groups was $25 \%$.

CONCLUSIONS: Increasing needle syringe program coverage and improving human immunodeficiency virus (HIV) knowledge are essential for efforts to eliminate inequalities in HIV risk behaviors among PWID.

KEY WORDS: Economics, Sexual behavior, Drug users, Cross-sectional studies, Iran

\section{Correspondence: Bahram Armoon}

Student Research Committee, School of Health, Shahid Beheshti University of Medical Sciences, Shahid Chamran Highway, Tehran 5511198353, Iran

E-mail: bahramarmun@gmail.com

Received: Sep 18, 2017 / Accepted: Nov 5, 2017 / Published: Nov 5, 2017

This article is available from: http://e-epih.org/

(C) This is an open-access article distributed under the terms of the Creative Commons Attribution License (http://creativecommons.org/licenses/by/4.0/), which permits unrestricted use, distribution, and reproduction in any medium, provided the original work is properly cited.

(C) 2017, Korean Society of Epidemiology

\section{INTRODUCTION}

It is estimated that 15.9 million (range, 11.0 to 21.2 million) people use drugs through injection globally [1], of whom 1.65 million are infected with human immunodeficiency virus (HIV) [2]. HIV infection among people who inject drugs (PWID) has been reported in more than 120 countries, and in many countries in Europe, Asia, and particularly in the Middle East, unsafe injection is the main mode of HIV transmission [1]. PWID are at increased risk of HIV infection due to both high-risk injecting and 
sexual practices [3]. In Iran, the PWID population size is estimated to be 170,000 to 230,000 people [4-6]. Risky sexual behaviors, such as having multiple partners, sex work, and unprotected intercourse, are common among PWID in Iran $[7,8]$. Unprotected sexual contacts in Iran were reported among more than $60 \%$ of PWID in their last sexual encounter with a commercial or casual partner [7]. This pattern of behavior allows HIV infection to spread within both injection and sexual networks $[9,10]$. A recent study of female partners of PWID in 3 cities in Iran showed the HIV prevalence to be as high as $2.8 \%$ among those who themselves did not inject drugs [7]. Many studies have assessed factors associated with risky sexual behavior among PWID $[7,11,12]$. Risky sexual behavior was significantly associated with HIV infection, the type of drug used, and the category of the partners [13-15]. Economic disparities in HIV-related high-risk behaviors among PWID remain [16]. Previous studies in some countries have shown risky sexual behaviors to be more prevalent in low economic status communities $[17,18]$. Low economic status and economic disparities may explain the differences in risky sexual behavior that cannot be explained by other individual or structural factors [18]. Lack of financial support and unstable economic status have been shown to be associated with high-risk behaviors, such as unprotected sex and sex work [19]. Previous studies have found economic disparities to play a role in HIV risk behavior, after adjusting multiple regression models for various observable characteristics, such as demographics and health status [18]. However, previous studies have not measured the degree to which these characteristics contribute to disparities. This knowledge is important for establishing and developing more effective HIV prevention programs. Moreover, the determinants of socioeconomic inequalities in access to and utilization of needle syringe programs (NSPs) have not been empirically investigated. To our knowledge, no previous study has systematically assessed the role of economic status in risky sexual behavior among PWID in Iran. In this study, we used Blinder-Oaxaca (BO) decomposition to explore the contribution of economic status to inequality in unprotected sex among PWID in Tehran and to decompose it into its determinants.

\section{MATERIALS AND METHODS}

Behavioral surveys among PWID were conducted in Tehran, the capital city of Iran, from November 2016 to April 2017. We employed a cross-sectional design and snowball sampling methodology. Eligible individuals were aged 18 years or older, reported injection drug use in the past month, were able to speak and comprehend Farsi enough to respond to the survey questions, and were able to provide informed consent. Study participants were recruited from street locations by snowball sampling. These participants were then given the opportunity to invite their peers to participate in the study. The participants recruited through snowball sampling also received coupons to distribute to their peers. The study procedures were reviewed and approved by the Ethical Committee of the University of Social Welfare and Rehabilitation
Sciences, Tehran, Iran. All data remained anonymous and confidential. The survey questionnaire used was a standardized behavioral questionnaire for PWID published by Family Health International [16]. The survey questionnaire included questions on socio-demographic characteristics, drug use and unprotected sex, access to harm reduction services, and HIV knowledge (low vs. high). The demographic variables assessed included age, marital status ( 0 , married or cohabitating; 1 , unmarried), highest level of education ( 0 , less than high school; 1 , high school diploma), homelessness in the past year $(0$, no; 1 , yes), methamphetamine use $(0$, no; 1 , yes) and history of incarceration ( 0 , no; 1 , yes ). HIV/acquired immune deficiency syndrome (AIDS) knowledge was measured with a 10-item set of questions covering basic knowledge of HIV/ AIDS, to which participants could answer 'yes,' 'no,' or 'don't know.

The outcome of interest was unprotected sex with any partner. Respondents were asked whether they had sex without a condom during last 6 months ( 0 , no; 1 , yes).

Our main covariate was economic status, which was assessed using principal component analysis (PCA). The data about socioeconomic status were gathered by a questionnaire that was developed in another study, entitled "Socio-economic status in Iran: a study of measurement index" [20]. The variables related to socioeconomic status in this questionnaire were educational status (high school or less vs. diploma or higher), participants' employment status (employed vs. unemployed), income status (monthly income), and housing (homelessness vs. stable housing), as well as items of convenience, such as owning their own car. Other covariates in our analysis included demographic and behavioral characteristics, such as age (years), age of first drug use ( $\geq 25$ years, $1 ;<25$ years, 0 ), age of first engaging in unprotected sex ( $\geq 25$ years, $1 ;<25$ years, 0 ), age of first drug injection ( $\geq 25$ years, $1 ;<25$ years, 0 ), HIV testing history, and marital status (single vs. married). HIV testing history was ascertained by the yes/no question 'Have you ever had an HIV test?' Having ever been tested was defined as having been tested for HIV and receiving the results at least once before the survey. All other behavioral questions referred to the 3 months prior to the interview.

We constructed the asset index (weighted by the first PCA factor) using socioeconomic data, and then divided the variable into 3 tertiles. The sum of score for the questionnaire was 48 . The first tertile was considered to be the high-income group and the third tertile to be the low-income group. We calculated the prevalence of unprotected sex among PWID with low and high incomes. The chi-square test was used to compare HIV-related high-risk sexual behaviors between the 2 PWID subgroups. We then used the BO method to decompose the economic inequality in unprotected sex. This involved decomposing the observed high-low economic gaps in the prevalence of unprotected sex into 2 components: composition and response effects. Composition effects represent the contribution of economic inequalities to unprotected sex due to economic differences in the distributions of observable HIV risk factors between the high and low groups (i.e., socio-demographic characteristics). Response effects reflect the contribution of eco- 
nomic inequalities in unprotected sex to economic differences in the effects of measured factors, as well as unmeasured factors not included in the model. To perform the decomposition, we used a logistic regression model with independent variables in each income group to determine the regression coefficient $(\beta)$ of each variable as the main effect and its interaction with the other independent variables. This method was based on 2 regression models, fitted separately for the 2 subgroups (i.e., the high-income and low-income groups). The analyses were performed in Stata version 11 (StataCorp., College Station, TX, USA) with an available Oaxaca package that supported the nonlinear decomposition for binary dependent variables proposed by Yun [19].

The BO decomposition method was introduced first by Blinder [21] and Oaxaca [22] to examine racial/gender discrimination in

Table 1. Overall characteristics of the sample of PWID, Tehran, 2016 $(\mathrm{n}=500)$

\begin{tabular}{|c|c|}
\hline Characteristics & $\mathrm{n}(\%)$ \\
\hline Age (mean $\pm S D, y r)$ & $33.45 \pm 9.7$ \\
\hline \multicolumn{2}{|l|}{ Education } \\
\hline Completed high school & $318(63.6)$ \\
\hline High school or less & $182(36.4)$ \\
\hline \multicolumn{2}{|l|}{ Living status } \\
\hline Homeless & $208(41.6)$ \\
\hline Stable housing & $292(58.4)$ \\
\hline \multicolumn{2}{|l|}{ Monthly income (USD) } \\
\hline$<150$ & $196(39.2)$ \\
\hline$\geq 150$ & $304(60.8)$ \\
\hline \multicolumn{2}{|l|}{ Age of first drug use } \\
\hline$<25$ & $260(52.0)$ \\
\hline$\geq 25$ & $240(48.0)$ \\
\hline \multicolumn{2}{|l|}{ Age of first drug injection } \\
\hline$<25$ & $245(49.0)$ \\
\hline$\geq 25$ & $255(51.0)$ \\
\hline \multicolumn{2}{|l|}{ Dropped out of school } \\
\hline Yes & $210(42.0)$ \\
\hline No & $290(58.0)$ \\
\hline \multicolumn{2}{|l|}{ Access to harm reduction programs } \\
\hline Yes & $227(45.4)$ \\
\hline No & $273(54.6)$ \\
\hline \multicolumn{2}{|l|}{ HIV knowledge } \\
\hline High & $355(71.0)$ \\
\hline Low & $145(29.0)$ \\
\hline \multicolumn{2}{|l|}{ Employment status } \\
\hline Unemployed & $352(70.4)$ \\
\hline Employed & $148(29.6)$ \\
\hline \multicolumn{2}{|l|}{ Marital status } \\
\hline Not married & $307(61.4)$ \\
\hline Married & 193 (38.6) \\
\hline Unprotected sex in the past $6 \mathrm{mo}$ & $200(40.2)$ \\
\hline
\end{tabular}

PWID, people who inject drugs; SD, standard deviation; USD, US dollar; HIV, human immunodeficiency virus. the labor market. The core idea is to explain the distribution of the outcome variable in question by a set of variables that vary systematically with socioeconomic status. The BO decomposition technique is especially useful for identifying and quantifying the separate contributions of group differences in measurable characteristics, such as education, experience, marital status, and geographical location, to racial and sex gaps in outcomes [23]. The aim of $\mathrm{BO}$ decomposition is to explain how much of the difference in mean outcomes between 2 groups is due to group differences in the levels of the explanatory variables, and how much is due to differences in the magnitude of the regression coefficients [23].

$\bar{Y}^{U}-\bar{Y}^{L}=\left[\sum_{i=1}^{N^{L}} \frac{\mathrm{F}\left(x_{1}^{L} \beta^{H}\right)}{N^{L}}-\sum_{i=1}^{N^{H}} \frac{F\left(x_{i}^{H} \beta^{H}\right)}{N^{H}}\right]+\left[\sum_{i=1}^{N^{L}} \frac{\mathrm{F}\left(x_{i}^{L} \beta^{L}\right)}{N^{L}}-\sum_{i=1}^{N^{L}} \frac{F\left(x_{i}^{L} \beta^{H}\right)}{N^{L}}\right]$

In equation (1), $\mathrm{N}$ refers to the sample size for the high-income and low-income groups. The first term in brackets shows the part of the risky sexual behavior gap that is attributable to differences in the distribution of characteristics (the explained component or endowments effect), and the second term represents the portion of the risky sexual behavior gap that is due to differences in the effects of these characteristics on risky sexual behaviors (the unexplained component or coefficient effect).

\section{RESULTS}

Out of the 520 recruited individuals, 20 were missing data for variables used to define their economic status, and were therefore excluded from the analysis. The study participants' age ranged from 19 to 67 years, with a median of 32 years (interquartile range, 24 to 38 years) (Table 1). Only $38.6 \%$ were married at the time of the study, and $36.4 \%$ had completed fewer than 6 years of educa-

Table 2. Factors associated with unprotected sex in subgroups of PWID defined by economic status

\begin{tabular}{lcc}
\hline & High-income & Low-income \\
\hline Age & $1.53(1.23,1.74)$ & $1.14(0.72,1.80)$ \\
Education & $1.00(1.54,1.34)$ & $0.92(0.52,1.80)$ \\
Living status & $1.48(1.26,1.84)$ & $2.91(1.88,4.50)$ \\
Marital status & $0.44(0.18,1.60)$ & $0.46(0.74,2.87)$ \\
Methamphetamine use & $1.15(0.74,1.27)$ & $1.20(0.71,1.90)$ \\
Dropped out of school & $1.48(0.90,2.44)$ & $1.23(0.71,1.92)$ \\
Age of first drug use & $1.12(0.72,1.80)$ & $1.42(0.74,2.85)$ \\
Age of first unprotected sex & $1.10(0.51,2.02)$ & $1.20(0.68,2.07)$ \\
Age of first drug injection & $0.31(0.05,2.10)$ & $0.92(0.52,1.74)$ \\
History of incarceration & $1.22(0.78,1.93)$ & $0.76(0.48,1.31)$ \\
Knowledge of partner's HIV status & $1.34(0.63,2.78)$ & $2.10(0.91,3.54)$ \\
HIV knowledge & $1.60(1.26,2.84)$ & $2.85(1.78,4.40)$ \\
Access to harm reduction programs & $2.70(2.05,3.74)$ & $1.30(0.40,2.78)$ \\
\hline
\end{tabular}

Values are presented as odds ratio ( $95 \%$ confidence interval). PWID, people who inject drugs; HIV, human immunodeficiency virus. 
tion. Of the participants, $60.8 \%$ reported a monthly income of 150 US dollars or more. Fifty-two percent had started drug use before the age of 25 years, and unprotected sex in the past 6 months was reported by $40.2 \%$ (Table 1 ).

Table 2 shows coefficient estimates (odds ratios [ORs]) between the income groups in multivariable logistic regression models. In the high-income group, PWID who were homeless (OR, 1.48; $\mathrm{p}=0.03)$, had low HIV knowledge (OR, 1.60; $\mathrm{p}=0.04)$, and had no access to harm reduction programs $(\mathrm{OR}, 2.70 ; \mathrm{p}=0.01)$ were more likely to have unprotected sex (Table 2). In the low-income group, PWID who were homeless (OR, 2.91; $\mathrm{p}=0.03$ ), had low HIV knowledge (OR, 2.85; $\mathrm{p}=0.02)$, and had no access to harm reduction programs $(\mathrm{OR}, 1.30 ; \mathrm{p}=0.30)$ were more likely to report unprotected sex. We found that the associations between a covariate and the dependent variable (unprotected sex) varied across income groups. For instance, the coefficient (OR) estimates for age of first drug use (1.12 vs. 1.42), HIV knowledge (1.60 vs. 2.85), and lack of access to harm reduction programs ( 2.70 vs. 1.30$)$ were considerably different between the high-income and low-income groups.

Table 3 presents a detailed decomposition of the disparity by the differences in the estimated coefficient (i.e., ORs) for each covariate in the logistic regression models summarized in Table 2. For instance, the estimated coefficient of not having access to harm reduction programs was lower in the low-income group (OR, 1.30 in Table 2) than in the high-income group (OR, 2.70), which led to an increase of the low-high disparity by 5.5 percentage points (the largest 'share' of the $21.4 \%$ discrepancy). The 'share' of the constant term indicates that out of the unadjusted total low-high disparity in unprotected sex, 53\% was still unexplained by the observable characteristics included in the regression model. The dif-

Table 3. Decomposition analysis of the contribution of socio-demographic factors to economic inequalities in unprotected sex

\begin{tabular}{|c|c|c|c|}
\hline Variables & Coefficient & $95 \% \mathrm{Cl}$ & Contribution (\%) \\
\hline Prevalence in high-income group & 0.152 & $0.640,0.150^{*}$ & \\
\hline Prevalence in low-income group & 0.402 & $0.162,0.310^{*}$ & \\
\hline Differences & 0.250 & $0.180,0.033^{*}$ & 100.00 \\
\hline Differences in characteristics & 0.123 & $0.082,-0.153$ & 47.00 \\
\hline Age & 0.001 & $-0.005,0.005$ & -0.12 \\
\hline Educational level & 0.019 & $0.002,0.036$ & 6.42 \\
\hline Marital status & 0.008 & $0.700,-2.300$ & 2.29 \\
\hline Age of first unprotected sex & -0.003 & $-0.007,0.001$ & -1.04 \\
\hline Knowledge of partner's HIV status & -0.004 & $-0.012,0.004$ & -0.54 \\
\hline Dropping out of school & $<0.001$ & $-0.005,0.005$ & 0.90 \\
\hline Age of first drug use & 0.003 & $-0.007,0.014$ & 1.34 \\
\hline Age of first injection & -0.001 & $-0.009,0.006$ & 0.54 \\
\hline History of incarceration & -0.005 & $-0.020,0.010$ & -2.95 \\
\hline Access to harm reduction programs & 0.055 & $0.032,0.078$ & 21.40 \\
\hline HIV/AIDS knowledge & 0.040 & $0.023,0.058$ & 13.56 \\
\hline Methamphetamine use & 0.018 & $0.007,0.029$ & 7.50 \\
\hline HIV testing & -0.001 & $-0.003,0.001$ & -0.22 \\
\hline Due to difference in coefficients (odds ratios) & 0.134 & $0.068,0.201$ & 53.00 \\
\hline Age & 0.006 & $-0.007,0.019$ & 2.36 \\
\hline Educational level & -0.001 & $-0.011,0.010^{*}$ & -0.21 \\
\hline Marital status & 0.003 & $-0.019,0.025$ & 1.20 \\
\hline Dropping out of school & 0.002 & $-0.012,0.017$ & 0.87 \\
\hline Age of first drug use & 0.220 & $-0.019,0.063$ & 8.43 \\
\hline Age of first injection & -0.001 & $-0.011,0.010$ & 0.21 \\
\hline Age of first unprotected sex & -0.013 & $-0.041,0.015$ & -5.20 \\
\hline Access to harm reduction programs & -0.067 & $-0.215,0.081$ & -26.60 \\
\hline HIV/AIDS knowledge & -0.040 & $-0.243,0.145$ & -18.89 \\
\hline Knowing partner's HIV status & 0.110 & $-0.036,0.256$ & -42.70 \\
\hline Methamphetamine use & -0.104 & $-0.224,0.016$ & -40.35 \\
\hline History of incarceration & -0.001 & $-0.011,0.010$ & 0.23 \\
\hline HIV testing & 0.011 & $-0.082,0.105$ & 4.37 \\
\hline Constant & 0.70 & $0.01,1.50$ & 75.70 \\
\hline
\end{tabular}

$\mathrm{Cl}$, confidence interval; HIV, human immunodeficiency virus; AIDS, acquired immune deficiency syndrome. ${ }^{*} p<0.05$. 
ference in the prevalence of unprotected sex between the high-income and low-income groups was $25 \%$. The gap between the lowincome and high-income groups was decomposed into its components. The decomposition analyses indicated that selected socio-demographic factors jointly explained a large proportion of the inequalities in unprotected sex among PWID. The selected predictor variables (age, education level, marital status, age of first engaging in unprotected sex, knowledge of partner's HIV status, dropping out of school, age of first drug use, age of first injection, history of incarceration, access to harm reduction programs, HIV/ AIDS knowledge, and methamphetamine use) together explained $47 \%$ ( 12 percentage points of the $25 \%$ gap) of the total inequality in unprotected sex, and the remaining 13 percentage points constituted the unexplained residual. Access to harm reduction programs made the largest contribution to the total inequality in unprotected sex among PWID. These results imply that the high-low disparity in unprotected sex could be reduced by only $47 \%$ even if the 2 groups became equivalent in all the covariates in the regression model. Moreover, HIV/AIDS knowledge and methamphetamine use accounted for about 13.5 and $7.5 \%$ of the total health inequality, respectively. The remaining gap was due to differences in the effects of the variables studied, as well as other factors that were not included in this study (unexplained components or coefficient effects). This may be attributed to factors correlated with economic status that were not included.

\section{DISCUSSION}

In the present study, we estimated the absolute difference in unprotected sex in PWID in Tehran, comparing those with a low economic status to those with a high economic status, and identified sources of economic inequalities. The decomposition analysis showed that only about $47 \%$ of disparities in unprotected sex among PWID could be reduced by equalizing commonly observable characteristics in these 2 income groups. In this study, we found that PWID who had a low economic status were more likely to have unprotected sex than PWID who had a high economic status. These findings align with those of studies that have found lower socioeconomic status to be associated with HIV risk behaviors, such as unprotected sex, in PWID [24-27]. Many studies using regression models have shown a correlation between socioeconomic status and unprotected sex among PWID [25,28, 29]. The results of this study showed that access to harm reduction programs was a major contributor to economic inequalities in unprotected sex. Overall, these factors explained $22 \%$ (5.5 percentage points of the $25 \%$ gap) of the economic inequalities among PWID in Tehran. This is consistent with previous international studies [30,31]. These findings emphasize the importance of targeted prevention programs, such as harm reduction programs, for sexual risk behaviors. The results of this study showed that HIV/AIDS knowledge played an important role in economic inequalities in unprotected sex, in accordance with a previous study conducted in China and the results of a review of interna- tional evidence [30,31]. Nazari et al. [26] found a negative association between HIV knowledge and unprotected sex, and reported that higher HIV knowledge levels could reduce unprotected sex among PWID. Hajebi et al. [31] indicated that adding a skillbased HIV prevention psychoeducation program to NSPs effectively reduced high-risk sexual behaviors among the clients of 2 drop-in centers in Tehran. Our findings suggest that striving to promote HIV knowledge in lower income groups might be effective in buffering the effects of economic inequality. Thus, HIV prevention programs should strongly focus on promoting HIV knowledge among their clients. Policymakers should pay more attention to implementing and expanding education programs on HIV at drop-in centers for PWID. Moreover, based on the results of our study, the contribution of economic inequalities to sexual risk behaviors was partially explained by other factors. This is consistent with the results of a previous study conducted in 21 countries in sub-Saharan Africa [17]. Using the BO approach, we found that socio-demographic characteristics partly explained the economic inequality in high-risk sexual behaviors among PWID in Tehran. Our findings showed that participants' educational level could explain inequalities in sexual risk behaviors. This is in accordance with a recent study by Chikovani et al. [13] that demonstrated that a higher education level was negatively associated with sexual risk behaviors in Georgia.

There were several limitations to this study. The first major limitation was its cross-sectional design, because it was not possible for us to investigate causal relationships directly. Therefore, a longitudinal study is required. Furthermore, our data were based on participants' self-reports, making our findings potentially subject to recall and social desirability bias [33]. Third, the sample was not random, and participants were recruited using snowball sampling techniques, which may have biased the sample because of the size of participants' social networks and homophily in recruitment patterns. Caution is therefore necessary in generalizing our results to all PWIDs living in Iran. The determinants of economic inequalities in HIV risk behaviors are poorly understood. Sociodemographic factors have been found to contribute to differences in HIV risk behaviors between low- and high-income groups [17].

In conclusion, the contribution of economic inequalities to highrisk sexual behaviors was primarily explained by the differential effects of access to NSPs and HIV knowledge among PWID. Increasing NSP coverage and improving HIV knowledge are therefore essential for efforts to eliminate inequalities in HIV risk behaviors among PWID.

\section{ACKNOWLEDGEMENTS}

We gratefully thank all staff members in the drop-in centers in Tehran who contributed in to recruitment, data collection, and interviewing. We thank the participants for their time and interest in the study. 


\section{CONFLICT OF INTEREST}

The authors have no conflicts of interest to declare for this study.

\section{ORCID}

Mehdi Noroozi: https://orcid.org/0000-0002-6511-1591; Hamid Sharifi: https://orcid.org/0000-0002-9008-7618; Alireza Noroozi: https://orcid.org/0000-0002-5838-4348, Fatemah Rezaei: http://orcid.org/0000-0003-2977-2699; Mohammad Rafi Bazrafshan: http:// orcid.org/0000-0003-3655-7778; Bahram Armoon: http://orcid.org/ 0000-0001-5467-9889

\section{REFERENCES}

1. Mathers BM, Degenhardt L, Phillips B, Wiessing L, Hickman M, Strathdee SA, et al. Global epidemiology of injecting drug use and HIV among people who inject drugs: a systematic review. Lancet 2008;372:1733-1745.

2. Burns L. World Drug Report 2013 By United Nations Office on Drugs and Crime New York: United Nations, 2013ISBN: 978-92-1 -056168-6, 151 pp. Grey literature. Drug Alcohol Rev 2014;33: 216-216.

3. Aceijas C, Stimson GV, Hickman M, Rhodes T; United Nations Reference Group on HIV/AIDS Prevention and Care among IDU in Developing and Transitional Countries. Global overview of injecting drug use and HIV infection among injecting drug users. AIDS 2004;18:2295-2303.

4. Mirahmadizadeh AR, Majdzadeh R, Mohammad K, Forouzanfar $\mathrm{MH}$. Prevalence of HIV and hepatitis $\mathrm{C}$ virus infections and related behavioral determinants among injecting drug users of dropin centers in Iran. Iran Red Crescent Med J 2009;11:325.

5. Noroozi M, Mirzazadeh A, Noroozi A, Mehrabi Y, Hajebi A, Zamani $S$, et al. Client-level coverage of needle and syringe program and high-risk injection behaviors: a case study of people who inject drugs in Kermanshah, Iran. Addict Health 2015;7:164-173.

6. Vazirian M, Nassirimanesh B, Zamani S, Ono-Kihara M, Kihara M, Ravari SM, et al. Needle and syringe sharing practices of injecting drug users participating in an outreach HIV prevention program in Tehran, Iran: a cross-sectional study. Harm Reduct J 2005;2:19.

7. Sajadi L, Mirzazadeh A, Navadeh S, Osooli M, Khajehkazemi R, Gouya MM, et al. HIV prevalence and related risk behaviours among female sex workers in Iran: results of the national biobehavioural survey, 2010. Sex Transm Infect 2013;89 Suppl 3:iii37iii40.

8. Sharifi H, Mirzazadeh A, Noroozi A, Marshall BD, Farhoudian A, Higgs $\mathrm{P}$, et al. Patterns of HIV risks and related factors among people who inject drugs in Kermanshah, Iran: a latent class analysis. J Psychoactive Drugs 2017;49(1):69-73.

9. Alipour A, Haghdoost AA, Sajadi L, Zolala F. HIV prevalence and related risk behaviours among female partners of male injecting drugs users in Iran: results of a bio-behavioural survey, 2010.
Sex Transm Infect 2013;89 Suppl 3:iii41-iii44.

10. De P, Cox J, Boivin JF, Platt RW, Jolly AM. Social network-related risk factors for bloodborne virus infections among injection drug users receiving syringes through secondary exchange. J Urban Health 2008;85:77-89.

11. Niccolai LM, Shcherbakova IS, Toussova OV, Kozlov AP, Heimer $\mathrm{R}$. The potential for bridging of HIV transmission in the Russian Federation: sex risk behaviors and HIV prevalence among drug users (DUs) and their non-DU sex partners. J Urban Health 2009; 86 Suppl 1:131-143.

12. Somlai AM, Kelly JA, McAuliffe TL, Ksobiech K, Hackl KL. Predictors of HIV sexual risk behaviors in a community sample of injection drug-using men and women. AIDS Behav 2003;7:383393.

13. Chikovani I, Goguadze K, Bozicevic I, Rukhadze N, Gotsadze G. Determinants of risky sexual behavior among injecting drug users (IDUs) in Georgia. AIDS Behav 2013;17:1906-1913.

14. Armoon B, Noroozi M, Jorjoran Shushtari Z, Sharhani A, Ahounbar E, Karimi S, et al. Factors associated with HIV risk perception among people who inject drugs: findings from a cross-sectional behavioral survey in Kermanshah, Iran. J Subst Use 2017:1-4.

15. Noroozi M, Ahounbar E, Karimi SE, Ahmadi S, Najafi M, Bazrafshan A, et al. HIV risk perception and risky behavior among people who inject drugs in Kermanshah, Western Iran. Int J Behav Med 2017;24:613-618.

16. Amon J, Brown T, Hogle J, MacNeil J, Magnani R, Mills S, et al. Behavioral surveillance surveys (BSS): guidelines for repeated behavioral surveys in populations at risk of HIV. Arlington: Family Health International; 2000, p. 114-117.

17. Sia D, Onadja Y, Hajizadeh M, Heymann SJ, Brewer TF, Nandi A. What explains gender inequalities in HIV/AIDS prevalence in sub-Saharan Africa? Evidence from the demographic and health surveys. BMC Public Health 2016;16:1136.

18. Sia D, Onadja Y, Nandi A, Foro A, Brewer T. What lies behind gender inequalities in HIV/AIDS in sub-Saharan African countries: evidence from Kenya, Lesotho and Tanzania. Health Policy Plan 2014;29:938-949.

19. Yun MS. Decomposing differences in the first moment. Econ Lett 2004;82:275-280.

20. Garmaroudi GR, Moradi A. Socio-economic status in Iran: a study of measurement index. Payesh 2010;9:137-144 (Persian).

21. Blinder AS. Wage discrimination: reduced form and structural estimates. J Hum Resour 1973:436-455.

22. Oaxaca R. Male-female wage differentials in urban labor markets. Int Econ Rev 1973;14:693-709.

23. Fairlie RW. An extension of the Blinder-Oaxaca decomposition technique to logit and probit models. J Econ Soc Meas 2005;30: 305-316

24. Song Y, Li X, Zhang L, Fang X, Lin X, Liu Y, et al. HIV-testing behavior among young migrant men who have sex with men (MSM) in Beijing, China. AIDS Care 2011;23:179-186.

25. Li X, Wu G, Lu R, Feng L, Fan W, Xiao Y, et al. HIV-testing behavior and associated factors among MSM in Chongqing, China: 
results of 2 consecutive cross-sectional surveys from 2009 to 2010. Medicine (Baltimore) 2014;93:e124.

26. Nazari SS, Noroozi M, Soori H, Noroozi A, Mehrabi Y, Hajebi A, et al. The effect of on-site and outreach-based needle and syringe programs in people who inject drugs in Kermanshah, Iran. Int J Drug Policy 2016;27:127-131.

27. Lee MB, Wu Z, Rotheram-Borus MJ, Detels R, Guan J, Li L. HIVrelated stigma among market workers in China. Health Psychol 2005;24:435-438.

28. MacPhail C, Pettifor A, Moyo W, Rees H. Factors associated with HIV testing among sexually active South African youth aged 1524 years. AIDS Care 2009;21:456-467.

29. Zhang L, Xiao Y, Lu R, Wu G, Ding X, Qian HZ, et al. Predictors of HIV testing among men who have sex with men in a large Chinese city. Sex Transm Dis 2013;40:235-240.
30. Rhodes T, Singer M, Bourgois P, Friedman SR, Strathdee SA. The social structural production of HIV risk among injecting drug users. Soc Sci Med 2005;61:1026-1044.

31. Hajebi A, Naserbakht M, Noroozi A. Adding-on a brief skill-based HIV prevention psychoeducation to needle and syringe programs: a randomized controlled trial. Iran J Psychiatry Behav Sci 2016; 10:e7411.

32. Latkin CA, Vlahov D. Socially desirable response tendency as a correlate of accuracy of self-reported HIV serostatus for HIV seropositive injection drug users. Addiction 1998;93:1191-1197.

33. Noroozi A, Mirzazadeh A, Farhoudian A, Hajebi A, Khankeh HR, Higgs P, et al. Impact of HIV status notification on risk behaviors among men who inject drugs in Kermanshah, west of Iran. J Res Health Sci 2016;16:116-121. 